\title{
Vertical Fall of a Body Under Free Motion
}

\author{
Zdzisław Pluta, Tadeusz Hryniewicz* \\ Koszalin University of Technology, Raclawicka 15-17, PL 75-620 Koszalin, Poland \\ *E-mail address: Tadeusz.Hryniewicz@tu.koszalin.pl
}

„Rem, tene verba sequentur"

Katon the Elder

\begin{abstract}
The paper aims at the kinetics of a body under free fall, especially free body motion, the motion occurring in the Earth environs space, that is in the range of the gravity action. A new adequate theory of such a motion, opening new not known kinetic magnitudes, has been elaborated. This theory has its source, which is the dependence of the way length on time. That is the way characteristics created in this work. The worked out theory has been verified by experiment, referred to two different realities. One of them was the body motion in the air environment of the Earth environs space, with the second one occurring in a determined machining space-time. The adequate earth acceleration/gravity has been calculated taking advantage of the theory elaborated; and its value, as indicated, is equal $3.85 \mathrm{~m} \cdot \mathrm{s}^{-2}$. Two kinds of accelerations have been separated: inertial and gravitational. The relations occurring between their values on the neighbouring potential fields, unstable, and stable, have been discovered. That discovery indicates on the existence of quantum nature of gravitation.
\end{abstract}

Keywords: Material body; Kinetics; Free fall; Path/way length; Velocity; Acceleration; Inertia acceleration; Gravitation acceleration; Time constant; Way constant; Gravitation number constant

\section{INTRODUCTION}

Free fall, vertical throw, horizontal throw, oblique throw - they are particular types of the body free motion, the motion occurring in the Earth environs space, so in the range of acting the phenomenon called the gravitation. That non-material junction, connecting the body with Earth makes its flight/motion is always ended on this planet.

It is obvious, however, that further actions connected with the course of the path/way length on time are not determined univocally and require further analyses. That is of importance, especially in reference to the oblique throw, as it affects the configuration of the flight trajectory. That quest concerns also horizontal throw, but it seems to be less problematic here. Anyway, it should be analysed deeply, because the existent knowledge in this subject is still far from adequate, with multiple doubts and flaws, and does not reflects sufficiently the considered reality. 
This work has been devoted to one of these four types of motion, namely the free fall. Perspective attempts/purposes of the authors are regarded also to further three kinds of free motion. They are to be considered successively.

The up-to-date knowledge on the free fall of a body assumes that, omitting the resistance of environment, the only reaction of the terrestrial globe has been considered, with the gravity force as a measure. Even the latest handbook literature [1] also interprets this phenomenon the same way, not introducing any corrections to the theory. Thus still the assumption is made in the science that the following differential equation of the second order:

$$
a=\frac{d^{2} s}{d t^{2}}=g
$$

describes the kinetics of a body in the free fall. One may notice that there is an equality made between the acceleration $a$ of the body and the Earth acceleration, that is the gravity $g$. Both these accelerations, de facto equalizing the accelerations, are the second derivative of the path/way length $s$ against time $t$.

Further mathematical action is commonly known. Integrating this equation once, one obtains a linear dependence of the velocity $v$ on time $t$; double integrating of this equation gives a parabolic dependence of the path/way length $s$ on the independent variable $t$.

There are only three kinetic magnitudes $\langle g, v, s\rangle$ in this kinetic chain, whereas the initial link of this chain is the Earth acceleration $g$, which corresponds to the acceleration $a$ of the falling material body. Introduced here the symbolism means additionally that the threeelement sequence of the kinetic magnitudes is double-sided closed. That means at present the science does not have the theory which enables a broader description of the free fall phenomenon. At the beginning, there is the acceleration at the source of cognition way, which seems to be erroneous and is not justified physically.

The authors of this work maintain, that the body path/way length in its free fall varies exponentially at the function of time, or to say more strictly, this is an exponential curve rising progressively. That thesis is to be proven, with the proof being both theoretical and experimental in nature.

\section{GENERAL DESCRIPTION OF A BODY KINETICS UNDER FREE FALL}

At the source of the cognitive way of all physical phenomena, which occur with variable rate (intensity) or velocity between the neighbouring energetic states, there is a general differential description, namely

$$
d Z= \pm \frac{\partial Z}{\partial N} d N
$$

where: $d Z$ - total differential of magnitudes being dependent variables, $d N$ - total differential of magnitudes being independent variables, $\partial Z / \partial N$ - partial derivative of magnitudes being dependent against independent ones. The signs $( \pm)$ are the algebraic operators fulfilling a determined function. The sign $(+)$ just confirms the physical sense of the determined mathematical record, whereas the sign (-) ascribes a physical sense to this record. 
That general source differential equation has been presented in many previous Authors' works [2-7] with the monograph [2], where it has been referred to particular realities, specifically technological, having various initial conditions. These conditions, properly understood initial conditions, determine detailed solution of this type of equation. The matter concerns the initial conditions of a phenomenon or process and not these connected with the beginning of measure of determined physical magnitudes. Measurement of these magnitudes is a necessary condition but it is not sufficient. A sufficient condition is turned on the beginning of their measurements at the beginning of the phenomenon.

Recording of the equation (2), applied to the considered phenomenon, possesses the following configuration:

$$
d s= \pm \frac{\partial s}{\partial t} d t
$$

where: $d s$ - total differential of the path/way length, $d t$ - total differential of time, $\partial s / \partial t-$ partial derivative of the path/way length against time. For the considered phenomenon the sign $(+)$ has been referred to, then

$$
d s=\frac{\partial s}{\partial t} d t
$$

Now one may consider the path/way characteristics of the free fall of a body, by regarding the initial conditions of this kind of motion. It is worth noting that an initial kinetic magnitude being the path/way length is now at the source of cognitive way. That magnitude, expressed as the function of time, forms an adequate reference to further kinetic physical magnitudes, being the first, second, third, and further derivatives against time, respectively. The sequence of these magnitudes is right-sided open, and covers not only these known three magnitudes: the path/way length $s$, velocity $v$, and acceleration $a$ (in the free fall and in the vertical throw aliased/identified with the gravity $g$ ).

\section{CREATION OF PATH/WAY CHARACTERISTICS}

The path/way characteristics, which is the dependence of the path/way length on time, is created through the proper detailing of the equation (4). First, however, it is worth noting that it concerns (described by the mentioned equation) the accelerated body motion; further phases of the free fall have been omitted in these considerations, though they also occur characterizing with this the vertical vibrating free motion. Only the first phase of this complex motion has been presented, to explain it entirely. The attention was paid to confront the introduced here adequate description of this phase of motion with the existent descriptions which say de facto generally of the free fall regarding only a fragment of the body kinetics where it moves with an accelerated motion.

The primary zero position of a material body (Fig. 1) corresponds with its position on the time unstable static potential field $(A S P F)$, with the field containing both the time way unstable static potential field $(A S P F)_{s}^{0}$ and time unstable static potential field $(A S P F)_{t}^{0}$. That is a zero position of the body; liberating the action of external stimulus results in falling the 
body down with the accelerated motion towards the Earth. That kind of motion takes place until the moment when the body reaches next, the first position, where it will have now an instantaneous stable energetic state. The following potential fields: the instantaneous stable static way potential field $(S S P F)_{s}^{1}$ and instantaneous time field of that kind, i.e. $(S S P F)_{t}^{1}$, are related to this state. These neighbouring fields dividing the space-time have been presented as a dotted field in Fig. 1.

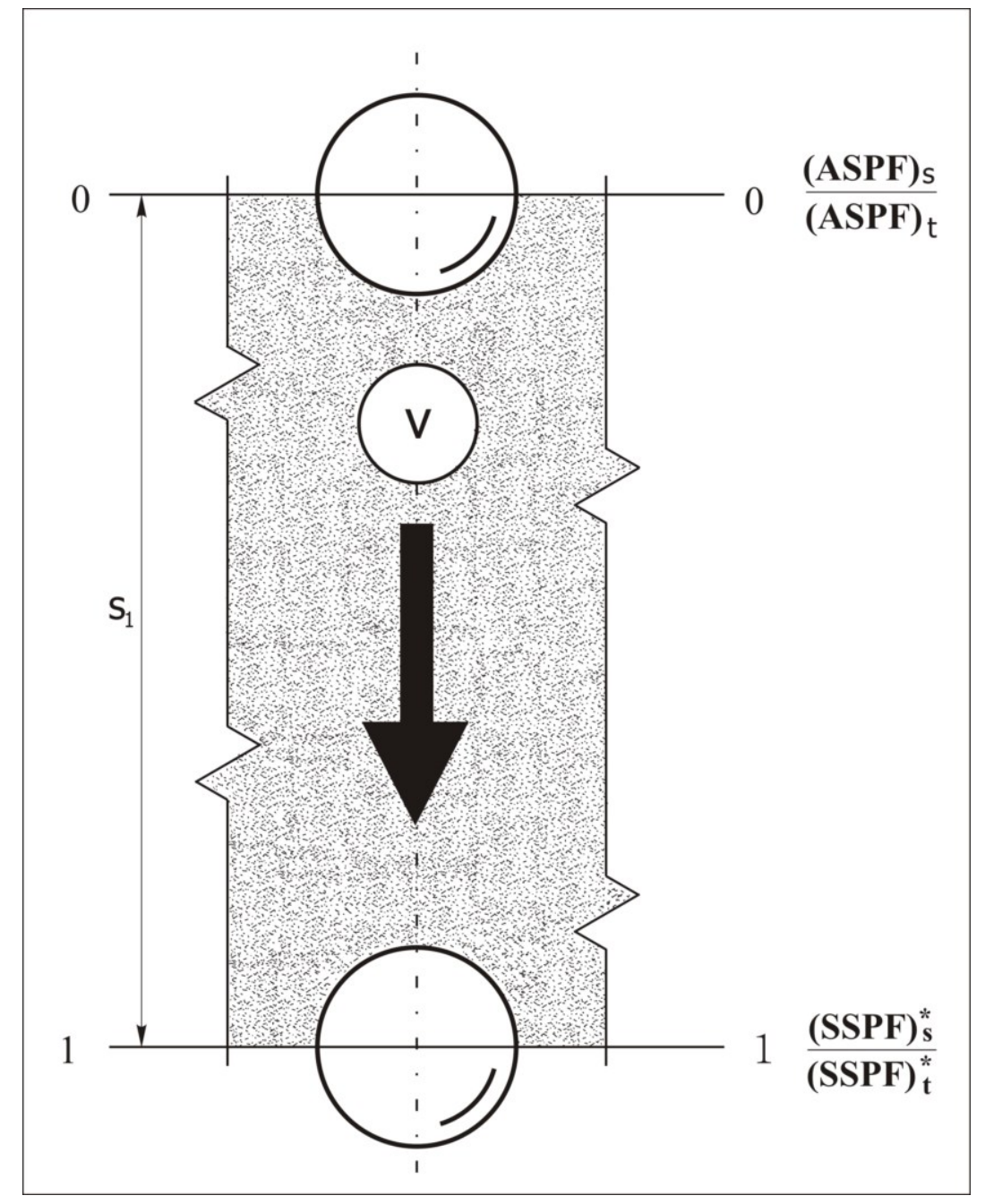

Fig. 1. Scheme of the material body position on the neighbouring potential fields

Now this space-time has been developed to present it on the background of the Ots coordinate system (Fig. 2). That made it possible to separate all four potential fields and present their positions in these two directions considered separately, that is the path/way direction $O s$ and time direction $O t$. 


\section{$s=s\left(e^{\frac{t}{e}}-1\right)$}

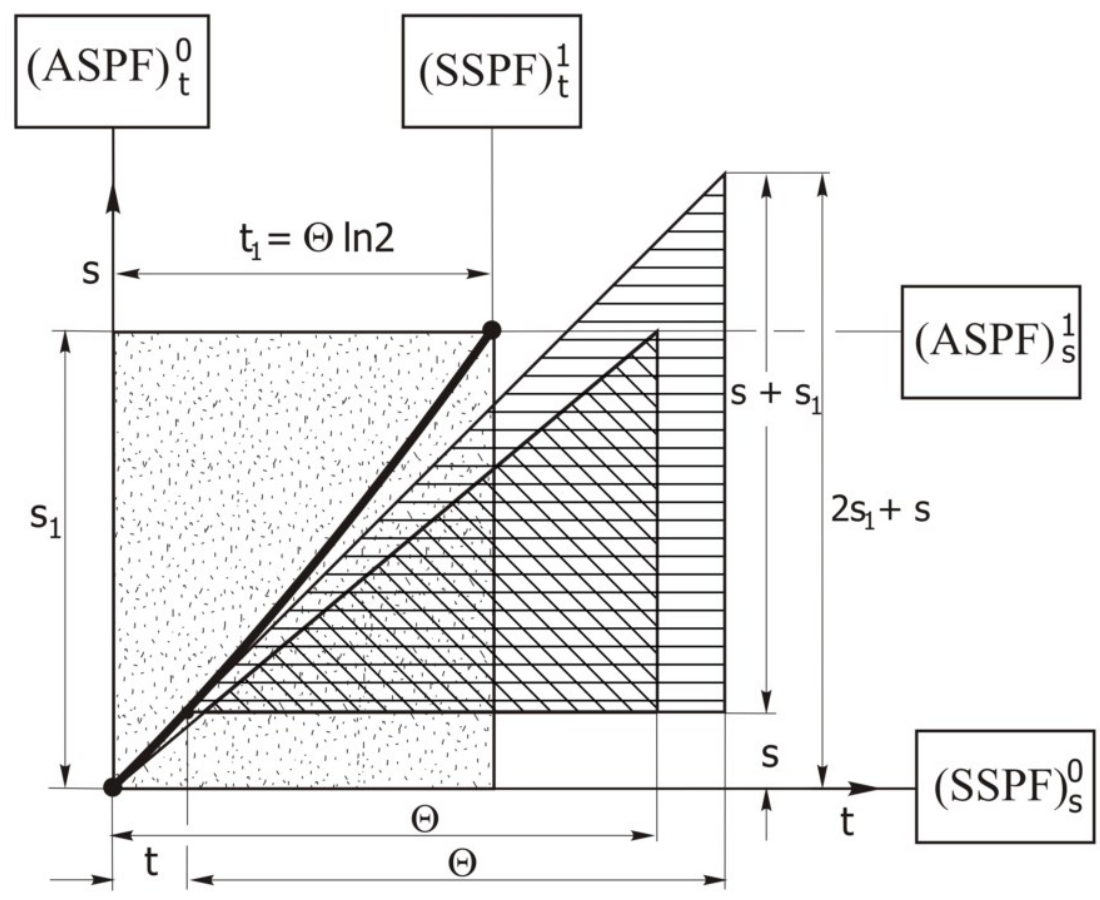

Fig. 2. Illustration of the scheme of creation of way characteristics

The path/way characteristics is reflected by progressively rising exponential curve. It forms an envelope of the right-angled triangles, with the time leg being constant and equaled the so called time constant $\Theta$, whereas the path/way leg of this triangle varies respectively. The initial point of the curve is on the intersection of the potential fields $(A S P F)_{t}^{0}$ and $(A S P F)_{s}^{0}$. The final point of this curve is situated on the intersection of the fields $(S S P F)_{t}^{1}$ and $(S S P F)_{s}^{1}$. The mentioned potential fields (as above described wider and developed in the contents) are the limits of space-time, with the dimension in the time direction as $t_{1}$ (to be closer determined further on), and in the path/way direction as $s_{1}$. The path/way leg of this shifting right-angled triangle is, as mentioned above, varying respectively, that results from the varying position of tangent to the path/way length curve. For $t+\Theta$ the length of this leg equals $s+s_{1}$. Now one may come to integrate the equation (4), denoting the limits of integrals and of total differentials. That means

$$
\int_{s}^{2 s+s_{1}} d s=\frac{\partial s}{\partial t} \int_{t}^{t+\Theta} d t
$$


and further

$$
s+s_{1}=\frac{d s}{d t} \Theta
$$

or

$$
\frac{d s}{s+s_{1}}=\frac{1}{\Theta} d t
$$

One may notice that the partial differential has been substituted by the quotient of total differentials. It could be done as the total differentials have been clearly determined by introducing the limits of the integrals.

Furthermore, by integrating both sides of the equation (7), one obtains the result

$$
\ln \left(s+s_{1}\right)=\frac{1}{\Theta} t+C^{*}
$$

that is

$$
s+S_{1}=e^{\frac{t}{\Theta}+C^{*}}=e^{C^{*}} \cdot e^{\frac{t}{\Theta}}=C e^{\frac{t}{\Theta}}
$$

After regarding that for $t=0$ the path/way length $s=0$, one obtains

$$
C=S_{1}
$$

and after substituting (10) to (9)

$$
s=s_{1}\left(e^{\frac{t}{\Theta}}-1\right)
$$

One may determine now the second coordinate of the point 1 , that is $t_{1}$ - time relating to the end of the phenomenon of free fall, corresponding with the accelerated motion of the material body. That result is obtained through the introducing the path/way length $s_{1}$ and time $t_{1}$ to the equation (11). Thus

$$
t_{1}=\Theta \ln 2
$$




\section{FURTHER DERIVATIVES OF KINETIC MAGNITUDES}

The derived path/way characteristics, given by the formula (11), is just that initial source characteristics, being the dependence of the path/way length $s$ on time $t$. That is the result of a proper adequate approach to the description of the body kinetics during its free fall. Now the entire series, non-limited right-sided series of derivative kinetic magnitudes, may be formed. It is enough to determine consecutive derivatives of the path/way length against time, that is the first, second, third, and further derivatives, obtaining with this the velocity $v$, acceleration $a$, impulse $i$, and next - the magnitudes which, for the time being, have a potential character and may also be introduced to the description of the natural reality in the future.

It is worth noting that the correctly understood impulse occurs here, being the third derivative of the path/way length against time, and the first derivative of the acceleration. As it was mentioned in $[8,9]$, that magnitude, called also the acceleration of the second degree, is used to the description of the phenomenon of jerk, the phenomenon which occurs during very fast feed motion of the determined assemblies of machine tools [10,11].

Thus the formulae on the velocity $v$, acceleration $a$, and impulse $i$ are formed in the following, quite a simple manner:

$$
\begin{gathered}
v=\frac{d s}{d t}=\frac{s_{1}}{\Theta} e^{\frac{t}{\Theta}}=v_{0} e^{\frac{t}{\Theta}} \\
a=\frac{d^{2} s}{d t^{2}}=\frac{d v}{d t}=\frac{s_{1}}{\Theta^{2}} e^{\frac{t}{\Theta}}=\frac{v_{0}}{\Theta} e^{\frac{t}{\Theta}}=a_{0} e^{\frac{t}{\Theta}} \\
i=\frac{d^{3} s}{d t^{3}}=\frac{d^{2} v}{d t^{2}}=\frac{d a}{d t}=\frac{s_{1}}{\Theta^{3}} e^{\frac{t}{\Theta}}=\frac{v_{0}}{\Theta^{2}} e^{\frac{t}{\Theta}}=\frac{a_{0}}{\Theta} e^{\frac{t}{\Theta}}=i_{0} e^{\frac{t}{\Theta}}
\end{gathered}
$$

It results that, for $t=0$ all these magnitudes are expressed by the dependences: $v=v_{0}$, $a=a_{0}, i=i_{0}$. For $t=t_{1}=\Theta \ln 2$, that is the time relating to the end of the considered stage of the free fall, the mentioned magnitudes determine the dependences of type, as follows: $v=v_{1}=2 v_{0} ; a=a_{1}=2 a_{0} ; i=i_{0}=2 i_{0}$. The double increase of the velocity growth/increment $v$, acceleration $a$, and the impulse $i$ may be noticed.

The graphical illustration of the courses $v=f(t), a=f(t), i=f(t)$, presented in Fig. 3 , is to underline all these magnitude relations.

The marked feature of the double increase/increment of derivative magnitudes is specific of the considered kind of variable motion. (It concerns also the forced variable motion of the accelerating nature.) 


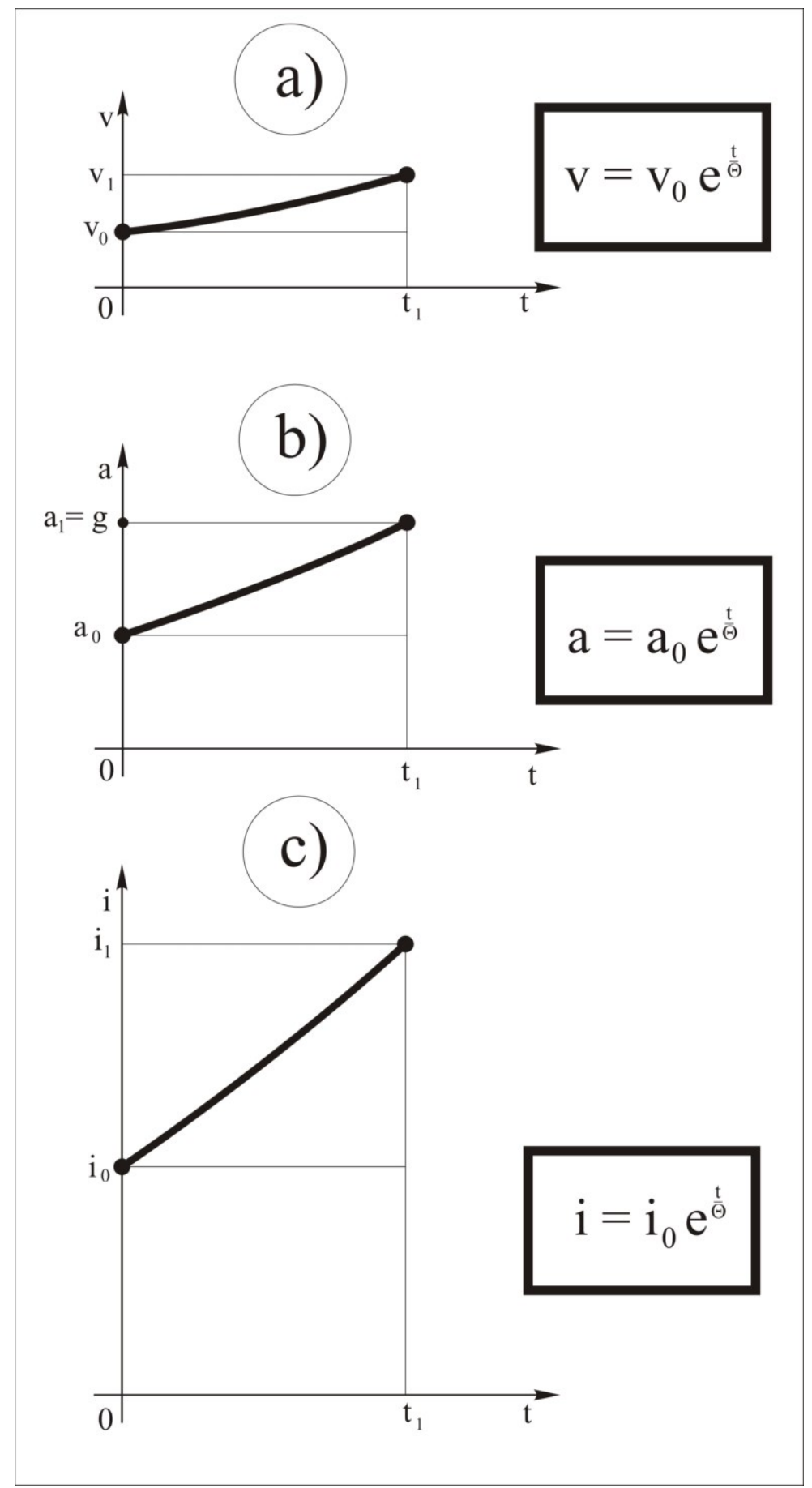

Fig. 3. Indicative courses of velocities (a), acceleration (b), and impulse (c) as the function of time

It is worth turning the attention on the curve of acceleration (Fig. 3b). As may be noticed, after the lapse of time $t=t_{1}$ it crosses the line relating to the Earth acceleration, that is the relation $a_{1}=g$ occurs in this point. It may be used to determine the values of gravity, by measure in advance the parameters of the space-time, namely the time $t_{1}$ and the path/way length $s_{1}$. 
Now, from the formula (12), the time constant should be determined, that is

$$
\Theta=\frac{t_{1}}{\ln 2}
$$

Taking advantage of the relation $a_{1}=2 a_{0}=g$, let us substitute (16) to (14), to obtain

$$
g=2 a_{0}=2 \frac{s_{1}}{\Theta^{2}}=\frac{2 s_{1}(\ln 2)^{2}}{\left(t_{1}\right)^{2}}
$$

or

$$
g=2(\ln 2)^{2}=0.96 \frac{s_{1}}{\left(t_{1}\right)^{2}}
$$

then

$$
g=0.96 \frac{s_{1}}{\left(t_{1}\right)^{2}}
$$

It is worth determining that adequate value of the acceleration. Data to this task have been excerpted from the literature [12]. There are three series of the measurements results of time $t_{1}$ and the path/way length $s_{1}$, with the time (as it occurs in square and requires to be measured more accurately) being a determined set of single results of measurement. Thus

1) $s_{1}=4.24 \mathrm{~m} ; t_{1}=1.0 ; 1.2 ; 1.1 ; 1.2 \mathrm{~s}$

2) $s_{1}=4.24 \mathrm{~m} ; t_{1}=1.1 ; 1.0 ; 1.1 ; 1.0 ; 1.0 \mathrm{~s}$

3) $s_{1}=8.72 \mathrm{~m} ; t_{1}=1.5 ; 1.3 ; 1.4 ; 1.4 ; 1.5 ; 1.3 \mathrm{~s}$

By substituting the values of the path/way length and the average values of particular time sets to the formula (19), three values of acceleration $g$ were obtained, so: $3.18 ; 4.07 ; 4.27$ $\mathrm{m} \cdot \mathrm{s}^{-2}$. The average value equals $3.85 \mathrm{~m} \cdot \mathrm{s}^{-2}$, that is

$$
g=3.85 \mathrm{~m} \cdot \mathrm{s}^{-2}
$$

That is not the end of verification of the existent theory, covering the description of kinetics of the body in the free fall. Furthermore, some other proofs will be presented to confirm the validity of the authors description of the phenomenon. It is to be confirmed using other means to prove the determined value of the gravity is right. 


\section{FREE FALL OF A BODY IN THE MACHINING TIME-SPACE}

The description of this specific machining process will allow to confirm the validity of the presented adequate theory by other method. That description of the process confirms the occurrence of the progressively rising path/way characteristics of the falling body and the resulting values of the Earth acceleration $g=3.85 \mathrm{~m} \cdot \mathrm{s}^{-2}$.

It will be about the external vertical free machining of material, that is such a case when the tool acts on a material from the side of open space (Fig. 4).

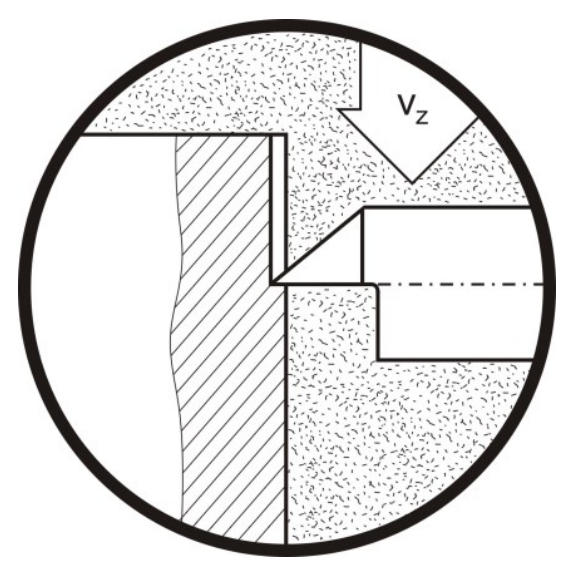

Fig. 4. Scheme of external vertical free machining of material

The trace resulting from that kind of treatment has a determined complex configuration (Fig. 5). At first the trace has the geometry of progressively rising exponential curve. It has been marked by a dotted area. In the point 1 the bending of that curve in the opposite direction occurs, and that means it has further exponential character, but it rises quite differently, just degressively.

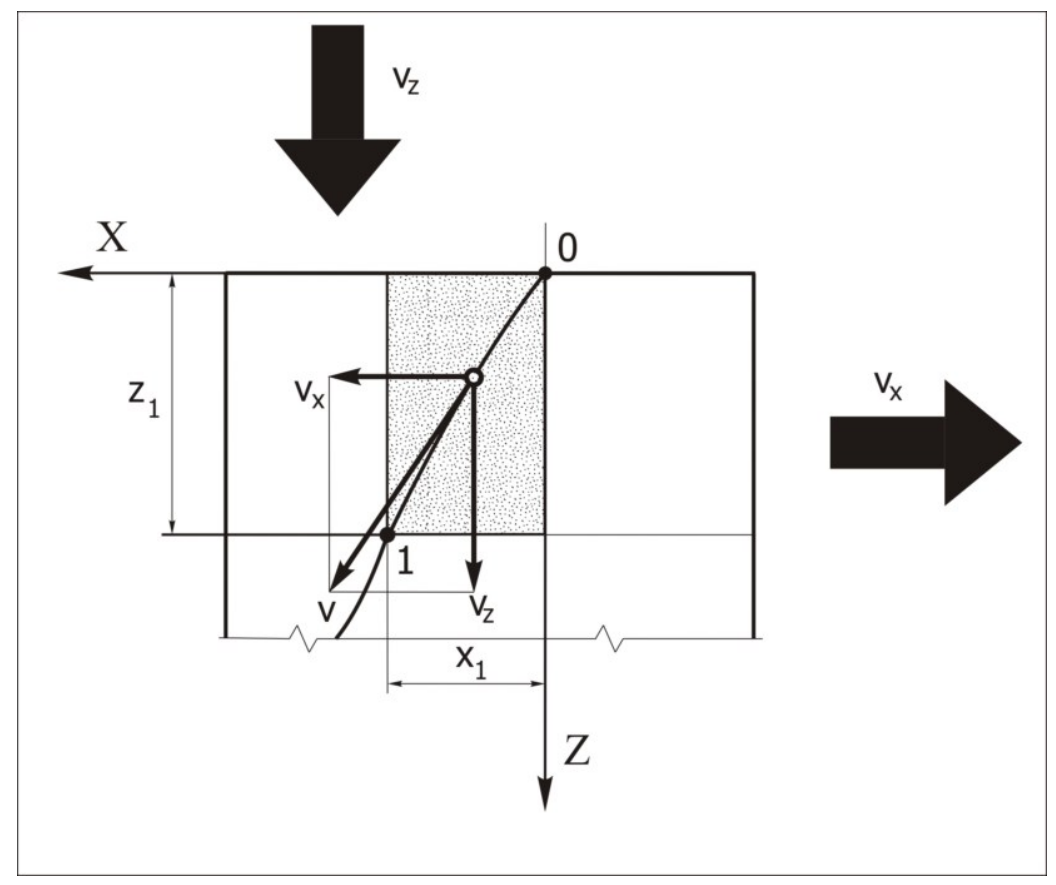

Fig. 5. Traces resulting from the vertical free machining of material at its horizontal velocity $v_{x}>0$ 
The basis of the reasons of that complex configuration of the machining trace is an adequately complex motion of the tool edge. (The subject of deeper analysis will be, as mentioned before, that first segment of the trace to be described more exactly.) Such a configuration of the first section of the trace results from the complex uniform motion of the workpiece and the accelerated free motion of the tool edge. Further formation of the trace is the result of superposition of the uniform workpiece motion and the retarded tool motion.

It is of great importance for description of the configuration of the machining trace to establish the position of coordinate system. It may look like a truism but it should be noticed herewith and consequently repeated: the beginning of the coordinate system must be connected with the beginning of the considered phenomenon. It is of absolute, first order condition to obtain the correctness of the description. Further part of this phenomenon will correspond with this phenomenon if the senses of axes of the system will be respectively oriented, that is in agreement with the senses of magnitudes characterizing this phenomenon. At this stage of consideration, it is about the velocity.

The beginning of the coordinate system corresponds with the beginning of the phenomenon of material treatment, so that system is a natural system of Cartesian rectangular coordinates. The axis $O X$ is on the height of upper free surface of the workpiece, and the axis $O Z$ covers/superimposes with the direction of tool action. The entire coordinate system moves together with the workpiece. The machining traces are described in that moving system. In such a coordinate system the sense of the axis $O Z$ corresponds with the sense of the tool velocity being the absolute velocity. The sense of the axis $O X$ in this moving system will be opposite to the sense of the velocity $v_{x}$ of the machined workpiece, then to the velocity of transportation; and opposite because the motion of this point of the machining zone (the tip of the tool edge) is considered with the relativity category that is quite natural for the motion. This point is moving away with the same velocity but running in opposite direction against the assumed moving system.

After those needed explanations one may write the equation of the constituent motions. They have the forms

$$
x=v_{x} \cdot t
$$

and

$$
z=z_{1}\left(e^{\frac{t}{T}}-1\right)
$$

After removal of time $t$ from both equations, one obtains

$$
z=z_{1}\left(e^{\frac{x}{v_{x} T}}-1\right)
$$

Here other symbolism of the time constant, namely denoted by a symbol $T$, was introduced; and it was done to stress its assignment to the other considered motion which is the complex motion. 
The product in a denominator of power of the number $e$, that is $v_{x} T$, is in turn the path/way constant $S_{x}$. It is interpreted (Fig. 6) as the workpiece path/way length, corresponding with the pass of tool to the neighbouring energetic state at an invariable constant initial intensity (rate) of the function increment $z=f(x)$.

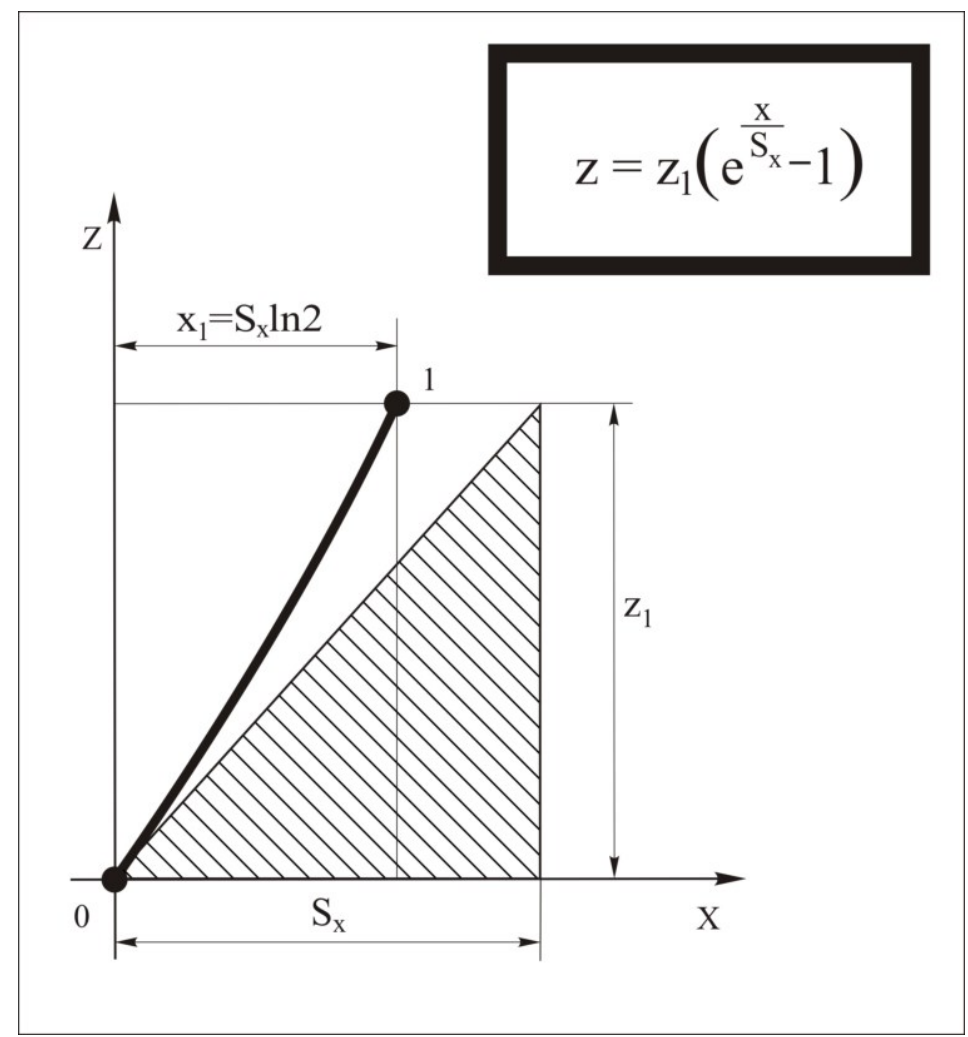

Fig. 6. Graphical and analytical interpretation of the contents of the dependence of the path/way length $z$ of tool on the path/way length $x$ of the machined workpiece

Regarding this introduced denotation, that latter function may be recorded as follows

$$
z=z_{1}\left(e^{\frac{x}{S_{x}}}-1\right)
$$

If $z=z_{1}$, then the second coordinate of the final point of the course, that is $x_{1}$, equals

$$
x_{1}=S_{x} \ln 2
$$

and that results from the formula (23) after substituting $z=z_{1}$ to it, and $x=x_{1}$.

Now let us consider the quantitative description of configuration of the machining trace formed in the material. Quite a complex, higher described treatment, was realized on the universal milling machine tool FWD 32J. The cutter guide was fixed in its holder/fixture by means of the adapter sleeve. A plate of perspex was machined by marking/scratching it from the side of the main surface after setting-up first the depth of cutter edge $(a=0.04 \mathrm{~mm})$ in the 
direction of the plate thickness. The machining was realized in the way that the edge was set in the free motion (including the cutter where this edge was fixed), and then it scratched the uniformly moving perspex plate. Afterwards that cutter was stopped on the slider flange placed in its bottom zone.

The velocity of the milling machine table, and with the same the plate velocity, i.e. $v_{x}$, was $6000 \mathrm{~mm} \cdot \mathrm{min}^{-1}$, that is $100 \mathrm{~mm} \cdot \mathrm{s}^{-1}$, or $0.1 \mathrm{~m} \cdot \mathrm{s}^{-1}$. The parameters of the machining trace had the following values: $z_{1}=50 \mathrm{~mm} ; x_{1}=11 \mathrm{~mm}$ (Fig. 7).

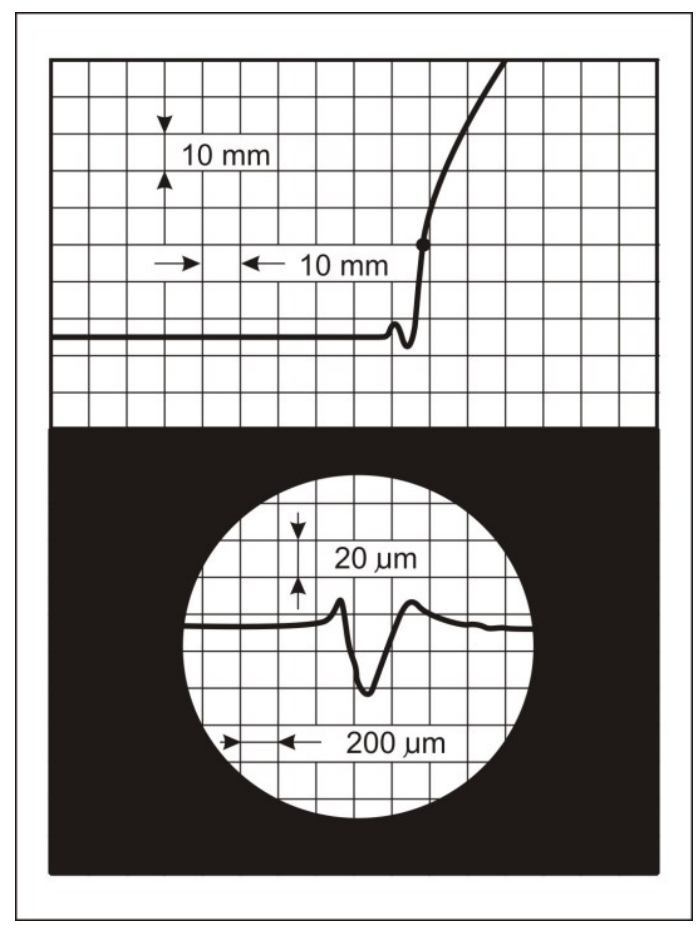

Fig. 7. Machining trace on the plate

Thus the path/way constant $S_{x}$, determined by the formula (24), and possessing the form

$$
S_{x}=\frac{x_{1}}{\ln 2}
$$

equals

$$
S_{x}=\frac{11}{0.693}=16 \mathrm{~mm}
$$

Therefore

$$
z=50\left(e^{\frac{x}{16}}-1\right)
$$


Now it is the time to describe this complex motion of the tool and the workpiece to determine an adequate value of the Earth acceleration in view of the confirmation of the above given value of gravity as the essential physical magnitude.

At this moment, the formula (19) will be used with the form adopted to the studied reality, with the following form:

$$
g=0.96 \frac{z_{1}}{\left(t_{1}\right)^{2}}
$$

The time $t_{1}$ will be determined from the formula (20), brought to the form

$$
t_{1}=\frac{x_{1}}{v_{x}}
$$

Substituting $x_{1}=11 \mathrm{~mm}=0.011 \mathrm{~m}$, and $v_{x}=0.1 \mathrm{~mm} \cdot \mathrm{s}^{-1}$, to the formula (26), one obtains

$$
t_{1}=\frac{0.011}{0.1}=0.11 \mathrm{~s}
$$

and following the formula (25) the calculated value of the time and measured value of the coordinate $z_{1}=50 \mathrm{~mm}=0.050 \mathrm{~m}$, one obtains the following searched value of the Earth acceleration/gravity:

$$
g=0.96 \frac{0.050}{(0.11)^{2}}=3.85 \mathrm{~m} \cdot \mathrm{s}^{-2}
$$

The constituent motions, that is the accelerated vertical motion and the uniform motion, may be described quantitatively too. The uniform motion, according to the formula (20), may be recorded as follows

$$
x=100 t, \mathrm{~mm}
$$

where $t$ in the formula (27) is given in seconds.

The accelerated vertical motion needs first the constant $T$ - to be calculated according to the formula (16). Substituting the symbol $\Theta$ by the introduced here the symbol, one obtains

$$
T=\frac{0.11}{\ln 2}=\frac{0.11}{0.693}=1.44 \mathrm{~s}
$$


and after introducing this value and $z_{1}=50 \mathrm{~mm}$ to the formula (21)

$$
z=50\left(e^{\frac{t}{1.44}}-1\right), \mathrm{mm}
$$

One may determine closer also the velocity $v_{z}$, then

$$
v_{z}=\frac{d z}{d t}=\frac{50}{1.44} e^{\frac{t}{1.44}}=34.72 e^{\frac{t}{1.44}}, \mathrm{~mm} \cdot \mathrm{s}^{-1}
$$

or, as indicated by the formula (13),

$v_{0}=34.72 \mathrm{~mm} \cdot \mathrm{s}^{-1}$, and $v_{1}=2 v_{0}=2 \cdot 34.72=69.44 \mathrm{~mm} \cdot \mathrm{s}^{-1}$.

It is also possible to determine further derivatives of magnitudes, such as the acceleration $a$, impulse $i$ and others, not named as yet the kinematic physical magnitudes.

\section{CONCLUSION}

In the summary it is worth noting that the introduced quite a new description of the kinetics of the body under its free fall is strictly adequate and respects the reality. It has been fully confirmed by the experimental verifications made. They showed that the free fall, when the body moves with the variable accelerated motion, has been described by the progressively rising exponential path/way characteristics, that is the dependence of the path/way length on time. That is the primary source characteristics of the body kinetics in the free fall. It does not close the series of further kinetic magnitudes but, contrary to the existent classical knowledge, with the only three-element set of such magnitudes, just opens it. Based on this primary dependence of the path/way length on time, one may create the time records of the velocity, acceleration, impulse, and further, yet not named the magnitudes though defined as the consecutive derivatives of the path/way length against time.

The precious discovery relies on that the acceleration of a body being the inertial acceleration is not equal to the Earth acceleration/gravity. Their equality exists only on the stable potential field where a stable equilibrium of the both material bodies, that is the considered body and the terrestrial globe, do.

That adequate analysis of the kinetics of a body in the free fall allowed to formulate a new essential law, the law of the Earth acceleration LEA: the Earth acceleration is in direct proportion to the path/way length of the free fall and reversely proportional to the time square of the body falling. Therefore

$$
g=k_{g} \frac{h_{1}}{\left(t_{1}\right)^{2}}
$$


where: $k_{g}$ - a number constant, equalled $0.96 ; h_{1}$ - the path/way length of the free fall; $t_{1}-$ the time of body falling.

It was proved that $g=3.85 \mathrm{~m} \cdot \mathrm{s}^{-2}$. Thus the adequate value of the Earth acceleration or the gravity is significantly less than the value resulting from the existent theory, possessing many cognitive flaws. This new theory has been verified experimentally so there is no doubt in it. Therefore, any decision made to reject it may mean presenting the position outside of it merits, which is non-scientific.

Separating two kinds of accelerations: inertial, and gravitational, as well as the discovery of relations between their values on the neighbouring potential fields: unstable, and stable, all that indicates the existence of quantum nature of gravity, with the existence of quanta: gravity (graviton) and inertia (inertion). That may be inspiring for the authors of sources [13-15] to change their creative activity to perform an adequate theory, describing the quantum nature of gravity. It is worth adding there is no need to present a quantum theory of gravity but simply the theory of quantum gravity. One cannot quantify the theory, anyway.

This subject is to be continued as very important for the science. One may get now to the dynamics of the body, which means its motion in the space-time, between the neighbouring potential fields or the sites related to the different energetic states of the material body, by taking into account of the body mass. That in turn necessitates providing an adequate definition of the energy. It is not the end of the series of new consecutive issues resulting from the presented cognitive quest. They will be the subject of the following works in a near future.

\section{References}

[1] J. R. Taylor, Classical mechanics, Vol. 1, Wydawnictwo Naukowe PWN SA, Warszawa 2006 (transl. from English), 1st Edition .

[2] Zdzisław Pluta, Fundamentals of surface smoothing using elastic wheels (in Polish), Publishing Koszalin University of Technology, Koszalin 2007.

[3] Zdzisław Pluta, Tadeusz Hryniewicz, International Letters of Chemistry, Physics and Astronomy 2 (2012) 28-34.

[4] Zdzisław Pluta, Tadeusz Hryniewicz, International Letters of Chemistry, Physics and Astronomy 3 (2012) 1-10.

[5] Zdzisław Pluta, Tadeusz Hryniewicz, International Letters of Chemistry, Physics and Astronomy 3 (2012) 11-23.

[6] Zdzisław Pluta, Tadeusz Hryniewicz, International Letters of Chemistry, Physics and Astronomy 4 (2012) 1-7.

[7] Zdzisław Pluta, Tadeusz Hryniewicz, International Letters of Chemistry, Physics and Astronomy 5 (2012) 35-45.

[8] H. Munirathnam, M. Kreis, Werkstatt und Betrieb 139 (2006) 42-50.

[9] K. E. Oczoś, Mechanik 5-6 (2007) 324-348.

[10] C. Zeppenfeld, F. Klocke, Werkstattechnik 94(6) (2004) 263-266.

[11] C. Zeppenfeld, F. Klocke, Maschinenmarkt 22 (2005) 32-33.

[12] M. Halaunbrenner, Practical exercises of physics, PWS, Warszawa 1968. 
[13] J. Magueijo, Faster than light, Publishing Amber, Sp. z o.o. Warszawa 2003, 1st Edition (transl. from English).

[14] P. Atkins, Galileo finger, Publishing Dom Wydawniczy Rebis, Poznań 2006 (transl. from English), 1st Edition.

[15] M. Chown, Quantum theory does not bite, Guide through the Universe, Publishing Zysk i Spółka, Poznań 2009 (transl. from English), 1st Edition. 